\title{
THE INTERCONNECTING MECHANISM FOR MONITORING REGULAR DOMESTIC CONDITION
}

\author{
A.L.Salunke ${ }^{1}$, Kanchan Kharat ${ }^{2}$, Dhanashree Rokade ${ }^{3}$, Poonam Sanap ${ }^{4}$ \\ ${ }^{1}$ Assistant Professor, Information Technology, BSIOTR, Maharashtra, India \\ ${ }^{2}$ Student, Information Technology, BSIOTR, Maharashtra, India \\ 3Student, Information Technology, BSIOTR, Maharashtra, India \\ 4Student, Information Technology, BSIOTR, Maharashtra, India
}

\begin{abstract}
In this paper we discuss about implementation of monitoring regular domestic condition using low cost sensing devices .The daily using small devices can make a communication with human being by using networking concept and cloud computing .Here we try to make machines intelligent so, they can tell about their state and requirements. So that taking care devices and operating them become more easy. In another words we can say that it is a new way of communication in which short range transreceivers are embedded in a daily using gadgets and forming communication between human and things, It makes machine more intelligent and providing them decision making quality.
\end{abstract}

Keywords: Internet of Things (IOT), Zigbee, Wireless Sensor network, Microcontroller, Energy Management, Smart Home.

\section{INTRODUCTION}

Moving towards the smart home we need some advanced system for our homes. For increasing communication between humans and machine personal home network is designed and it is also helpful in energy management. It is the new way of communication in which short range receiver and transmitter are embedded in small-small devices, daily gadgets for making intelligent network around the home and the peoples.

We know the several examples of smart homes such as GatorTech smart house, iDrom, GorgiaTech Aware Home. Till this date we know the many developments such as for commercial and safety purpose, Because this smart technology can protect us from many natural calamities and disasters. This system gives effective low cost and flexible solution for environmental condition monitoring and energy management in homes. The basic operation for energy manages is such as controlling ubiquitous devices like $\mathrm{AC}$, coffee machine, water cooler.

\subsection{Need}

The basic need of internet of things is communication, Control and automation, cost saving. The people who do not able to take care their appliances properly because of time they can communicate with their appliances by using Iot. They can control and automate devices by monitoring without manual checking. The electronic parts used in system are too cheaper and cost saving and so that it can affordable for all peoples.

\subsection{Basic Concepts}

The basic concept behind system is very practical and clear. The sensors are embed in the device/daily using appliances and a ADC(Analog to Digital Converter)is used that converts analog readings to digital sensed by sensors. MAX 232 is also here for communication

\section{SYSTEM ARCHITECTURE}

In Architecture we use client pc, cloud server, ADC, sensors, Controllers, Driver \& electronic devices. Local client pc connected with cloud server by using java networking. Pc links with sensors and electronic devices. Sensors convert temperature, pressure, speed, position and other data into either digital or analog electrical signals.

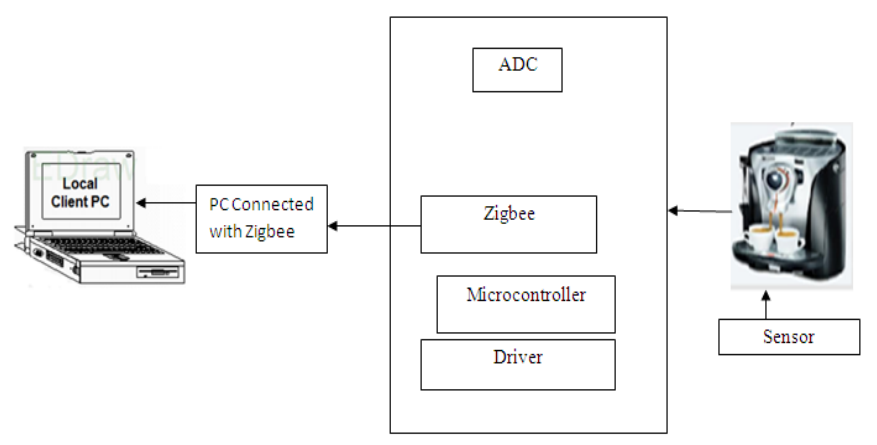

Fig-1: System Architecture 
ADC Stands for "Analog-to-Digital Converter." Since computers only process digital information, they require digital input. Therefore, if an analog input is sent to a computer, an analog-to-digital converter (ADC) is required. This device can take an analog signal, such as an electrical current, and digitize it into a binary format that the computer can understand.

A driver is software that allows your computer to communicate with hardware or devices.

A driver is a small piece of software that tells the operating system and other software how to communicate with a piece of hardware. A driver acts like a translator between the device and programs that use the device. A device that controls the transfer of data from a computer to a peripheral device and vice versa. For example, disk drives, display screens, keyboards, and printers all require controllers.

Zigbee is wireless network device. It is used for communication between two or more devices and it's range is 100 meters around it. In a ZigBee network, end devices collect and forward data to a coordinator and then ZigBee protocol data format is translated to Internet protocol (IPV6) format by the gateway.

The home automation scheme proposed in this paper can clearly measure electrical parameters and control domestic objects. The combined system will support the residents to avoid multiple systems to monitor their domestic consumption. The system can be run with the help of an resident favorite laptop or i-pad device. The basic layout shows key elements of the integrated WSN with internet system. It consists of Smart Sensing devices, IoT Gateway and Internet

\section{SYSTEM IMPLEMENTATION}

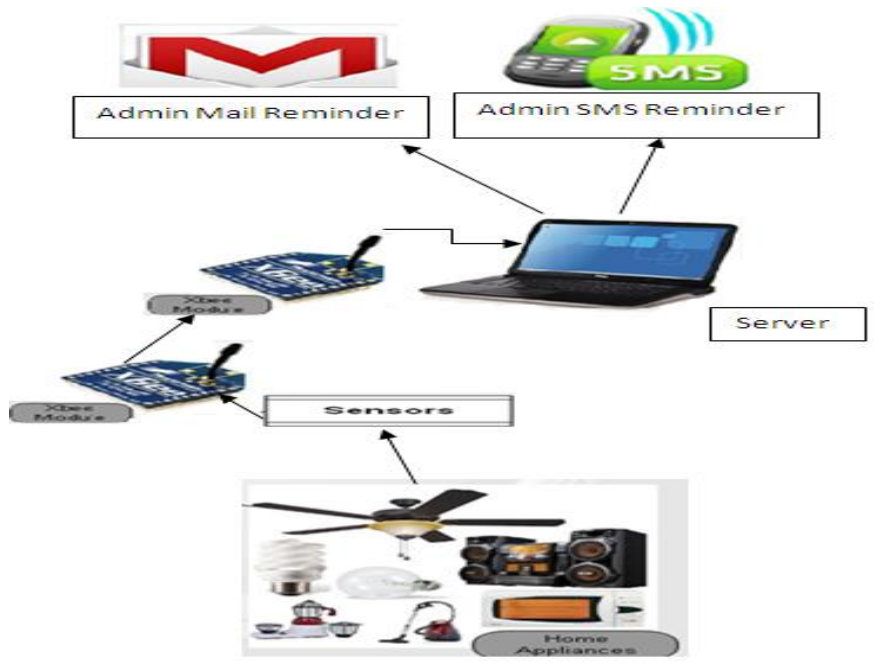

Fig-2: System Implementation
In figure 2 the actual system implementation is given so the main concept is that the server pc sends mail or sms to the admin or the authorized one if any deficiency or fault occurs. Sensors can get readings and all the operations get performed by the kit which consist ADC, Microcontroller and all electronic hardware mentioned in figure 1 . And via Zigbee it sends to server pc and server pc sends mail to the person which we have mentioned in system.

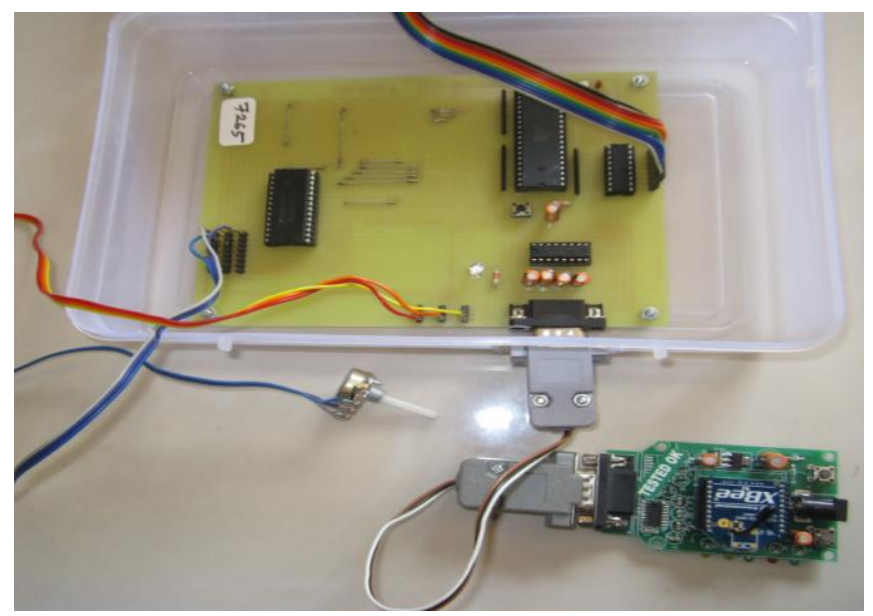

Fig-3: System internal structure

In figure 3 we can see the internal structure of the system where all the required hardware parts are embedded on a single chip and the power management is also done here. Max 232 is used for serial communication with pc. In above given kit at time we can connect 8 sensors at a time.

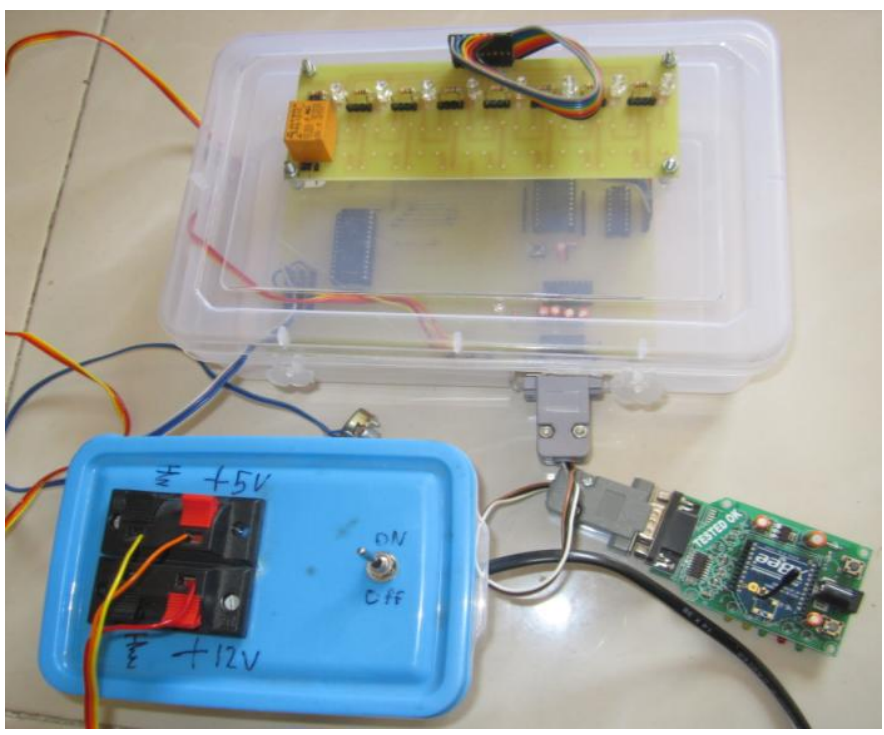

Fig-4: System with power supply

Here in figure 4 we can see how power management is done. A power management source is provided here for system 
safety, because of over flow of voltage the internal damage of hardware is occurs so that we have to connect capacitor and transformer to manage the power supply. Because of taking such precaution there is no danger of device failure.

\section{REQUIREMENT AND SPECIFICATION}

For designing such system we have some requirements such as required software and hardware some safety precautions to be take mentioned below-

\subsection{Hardware's Required}

In hardware requirement we want client pc and many primary devices some are explained below

\subsubsection{Zigbee}

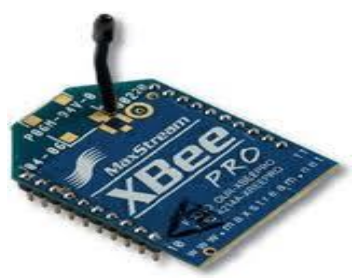

- Zigbee is a wireless networking device.

- It is a Hardware chip like structure.

- Used for wireless communication.

- It covers maximum of $100 \mathrm{~m}$ area around it.

\subsubsection{Microcontroller 89C51}

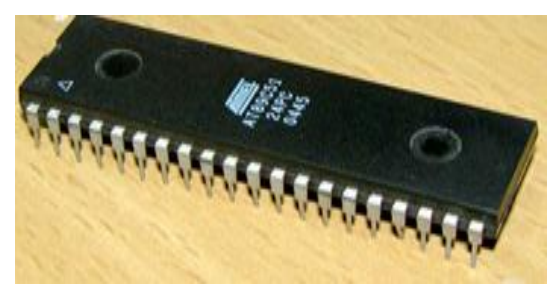

- $89 \mathrm{C} 51$ is an 8-bitMicrocontroller

- $\quad$ 89C51 has 4KB of Flash programmable and erasable read only memory (PEROM).

- 128 bytes of RAM.

- It can be erased and program to a Maximum of 1000 times.

\subsubsection{ADC 0880CCN}

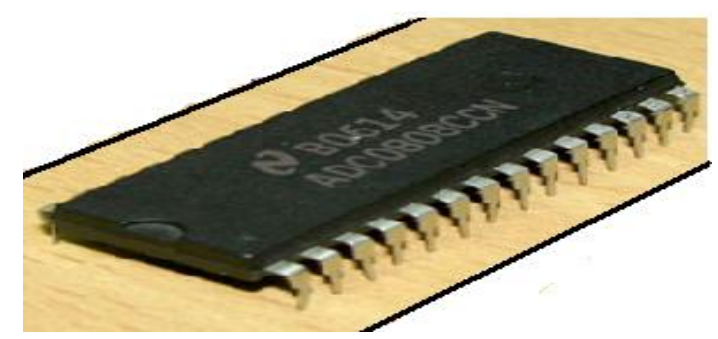

An analog-to-digital converter is a device that converts a continuous physical quantity (usually voltage) to a digital number that represents the quantity's amplitude.

\subsubsection{MAX 232}

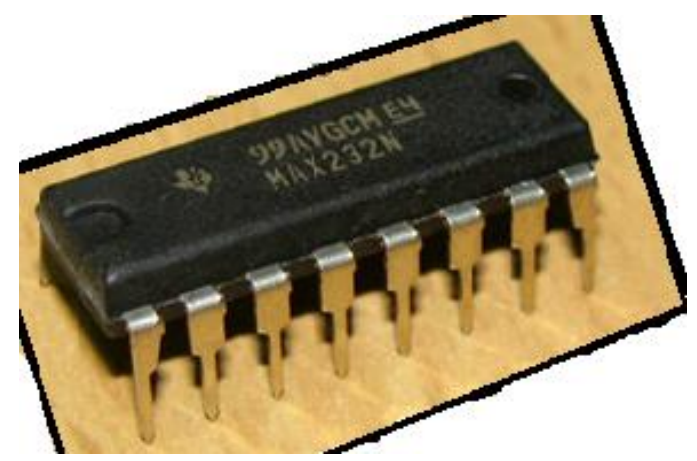

The MAX 232 IC is used to convert the TTL/CMOS logic levels to rs 232 logic levels during serial communication microcontroller with pc machine.

\subsubsection{Sensing Unit}

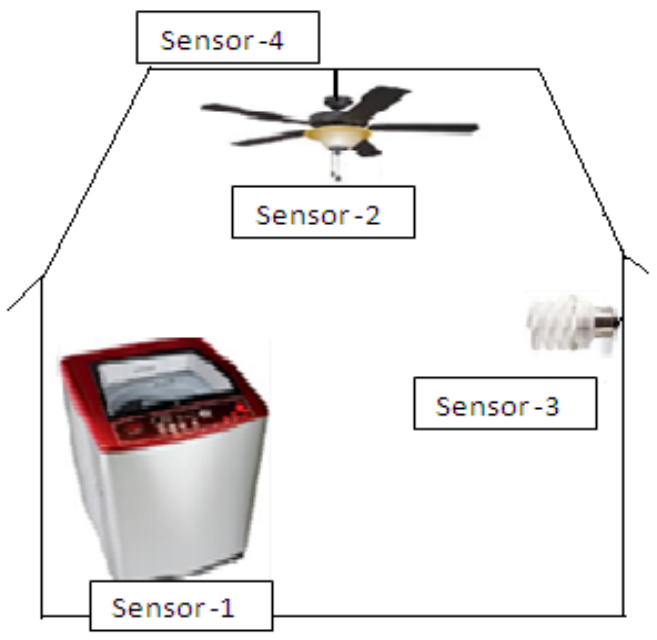

Fig-5: Home sensing unit

In figure 5 sensing unit is shown here we can use Different types of sensors for different devices.

The environmental parameters (temperature, humidity and light) are important aspects for deciding whether equipment such as (fans, electric heaters or lamps) should be switched on or off in a wireless monitoring network used for energy management in the home. The following sensors are used widely.

The sensor nodes used in the ZigBee WSN have a temperature sensor operating in the range of $-20^{\circ} \mathrm{C}$ to $+125^{\circ} \mathrm{C}$. The output voltage out of this sensor varies $1^{\circ} \mathrm{C}$ for every $10 \mathrm{mV}$ with 
$500 \mathrm{mV}$ offset voltage. The light sensor used here consists of a planar silicon NPN photodiode that is equipped with a flat glass window and built in colour correction filter designed to approximate the spectral response of the human eye. The AC voltage sensor has a range of 200-280Volts-RMS with an accuracy of \pm 2 Volts-RMS. The current sensor consisted of an ASM010 current transformer, with a range of 1 to $100 \mathrm{Amps}$ and operating temperature range from $-40^{\circ} \mathrm{C}$ to $+120^{\circ} \mathrm{C}$.

\subsection{Methodologies Used}

Here we see which technologies are used in the system implementation.

\subsubsection{Address Transformation}

The key element in the data transformation from ZigBee to IPV6 format is the address translation. This was implemented by the application gateway program for determining the source or destination address of a packet that encapsulates a ZigBee packets' payload. The corresponding application gateway performs the address transformation mechanism for ZigBee to address non ZigBee nodes. ZigBee is based upon the 802.14.5 protocol which uses a 64 bit address for each node on a PAN, and 16 bits to identify the PAN ID. IPv6 uses 128 bits to address a node on the network, of which 48 bits represent the network, 16 bits represent the local network (PAN ID), and 64 bits represent the host id (sensor node). Therefore, the node address for 802.15.4 can placed in an IPv6 address, and the PAN ID can be used to identify the ZigBee network in an IPv6 address.

\subsubsection{Packet Translation}

This is used for ZigBee packet transformation to IPv6 format.The packets originated from the XBee-S2 network are sent to a server using a tunneling technique, where the addressing information is removed and placed in the encapsulating protocol. Packets destined for the XBee-S2 network use a stateful translation where the source address is stored on the gateway. This enables reply packets from the XBee-S2 network to be sent to the correct address.

A serial interface is used to transmit Application Programming Interface (API) packets from/to the coordinator and router. The WRT54GL router has two serial ports - one of which is used to connect to the XBee-S2 coordinator. The router performs the conversion of the XBee-S2 API packet to an IPv6 packet.

\section{CONCLUSIONS}

In $21^{\text {st }}$ century of today the world is bided together with the help of internet. Internets have made world look small \& communication have become more effective \& easy global. It is expected that the internet ubiquitous. We have proposed a small home monitoring condition for communication, control $\&$ cost saving.

Our project is based on connecting of things for their better management. Internet of things is use to ease the human work of monitoring the things. Basically normal machine are not intelligent enough to make their own decision making concept. Now the theory which we have proposed will be having machines which are intelligent enough to make the basic decisions. Human intervention in this machine will be very less as compared to the normal machines \& still the machine will be able to carry out some task on its own. Here we are making the machine intelligent. We are providing it with decision making quality.

\section{ACKNOWLEDGEMENTS}

We take this opportunity to thank our project guide PROF.A.L.SALUNKE and Head of the Department PROF.P.A.BANDGAR for their valuable guidance and for providing all the necessary facilities, which were indispensable in the completion of this paper. We are also thankful to all the staff members of the INFORMATION TECHNOLOGY of BSIOTR, WAGHOLI.

We would also like to thank the institute for providing the required facilities, Internet access and important books.

\section{REFERENCES}

[1]. "The Internet of things", IEEE Wireless Communications, 2010, Vol.17, Issue.6, pp-8-9.

[2]. D. Surie, O. Laguionie, T. Pederson, "Wireless sensor networking of everyday objects in a smart homeenvironment", Proceedings of the International Conference on Intelligent Sensors, Sensor Networks and Information ProcessingISSNIP- 2008, pp. 189 - 194

[3]. Internet 3.0: The Internet of Things. (C) Analysys Mason Limited 2010

[4]. ZigBee Alliance, "Understanding ZigBee gateway", ZigBee Document 095465r13, September 2010.

\section{BIOGRAPHIES}

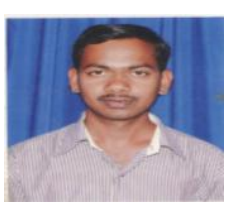

Appasab L. Salunke

Assistant professor in JSPM' BSIOTR(W)

College, IT Department, Wagholi ,Pune.

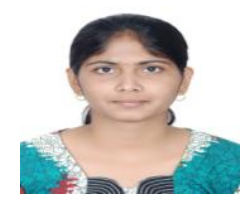

Kanchan Navnath Kharat Completing B.E. in JSPM's BSIOTR (W) College, IT Department, Wagholi Pune. 
Poonam Dattopant Sanap Completing B.E. in JSPM's BSIOTR (W) College, IT Department, Wagholi, Pune

Dhanashree Rokade Completing B.E. in JSPM's BSIOTR (W) College, IT Department, Wagholi, Pune. 